\title{
A Review of Diagnostic Methods for Invasive Fungal Diseases: Challenges and Perspectives
}

\author{
Diego R. Falci · Claudio M. B. Stadnik · Alessandro C. Pasqualotto
}

Received: February 3, 2017 / Published online: March 29, 2017

(C) The Author(s) 2017. This article is an open access publication

\begin{abstract}
Invasive fungal diseases are associated with a high morbidity and mortality, particularly in the context of immunosuppression. Diagnosis of invasive fungal diseases is usually complicated by factors such as poor clinical suspicion and unspecific clinical findings. Access to modern diagnostic tools is frequently limited in developing countries. Here, we describe five real-life clinical cases from a Brazilian tertiary hospital, in order to illustrate how to best select diagnostic tests in patients with different fungal infections.
\end{abstract}

Enhanced content To view enhanced content for this article go to http://www.medengine.com/Redeem/ A9F7F06018C7A9AA.

D. R. Falci

Hospital de Clinicas de Porto Alegre, Porto Alegre, Brazil

D. R. Falci

Centro Universitário La Salle Canoas, Canoas, Brazil

C. M. B. Stadnik - A. C. Pasqualotto ( $\square)$

Universidade Federal de Ciências da Saúde de Porto

Alegre, Porto Alegre, Brazil

e-mail: pasqualotto@santacasa.tche.br

C. M. B. Stadnik - A. C. Pasqualotto

Santa Casa de Misericordia de Porto Alegre,

Porto Alegre, Brazil
Keywords: Antigen detection; Beta-D-glucan; Galactomannan; MALDI-TOF; PCR

\section{INTRODUCTION}

Invasive fungal diseases (IFD) are important causes of mortality and morbidity in immunocompromised patients, including individuals suffering from hematological diseases, transplant recipients, and those under critical care or chemotherapy. Diagnosis of IFDs is complicated by several factors: tests based on antibody detection have low sensitivity, culture-based methods may require a long time to yield positive results, and biopsies for histopathologic examination can be hazardous for many of the severely ill patients. Finally, experience is needed from pathologists in order to perform a proper diagnosis, and biomarkers might be expensive and/or not widely available. Therefore, there is an urgent need to facilitate access to modern diagnostic tests, and also to wisely use current available diagnostic methods, aiming for an early initiation with appropriate antifungal therapy.

Here, we describe five real-life clinical situations of IFD in which diagnostic methods were required. All patients were selected in a reference transplant centre located in Porto Alegre, southern Brazil. The purpose of this review is to perform a practical approach by selecting cases from real life as examples of challenges related 
to the diagnosis of IFDs. In accordance, it should be noted that clinical decisions were made by the attending physicians, and these did not necessarily align with current international guidelines. Informed consent was obtained from all patients for being included in the study.

\section{CASE 1}

A 35-year-old female patient from Rondonia (a Brazil northern state) had been submitted to kidney transplantation 5 years ago. She was using tacrolimus and prednisone ( $2.5 \mathrm{mg}$ daily). She presented with a 2-month history of fever, night sweats and fatigue with no respiratory symptoms. A chest magnetic resonance imaging showed multiple bilateral pulmonary nodules, up to $2.5 \mathrm{~cm}$ in diameter (Fig. 1). She was put on empirical treatment for tuberculosis. Serum cryptococcal antigen (latex agglutination test) and Histoplasma immune diffusion test were negative. Bronchoscopy was performed and microscopic examination of bronchoalveolar lavage (BAL) fluid was negative for fungi. BAL galactomannan (GM) index was 0.3. An open lung biopsy was performed and revealed a granulocytic infiltrate, with spherical budding structures suggestive of Histoplasma capsulatum (small ovate yeast-like forms at

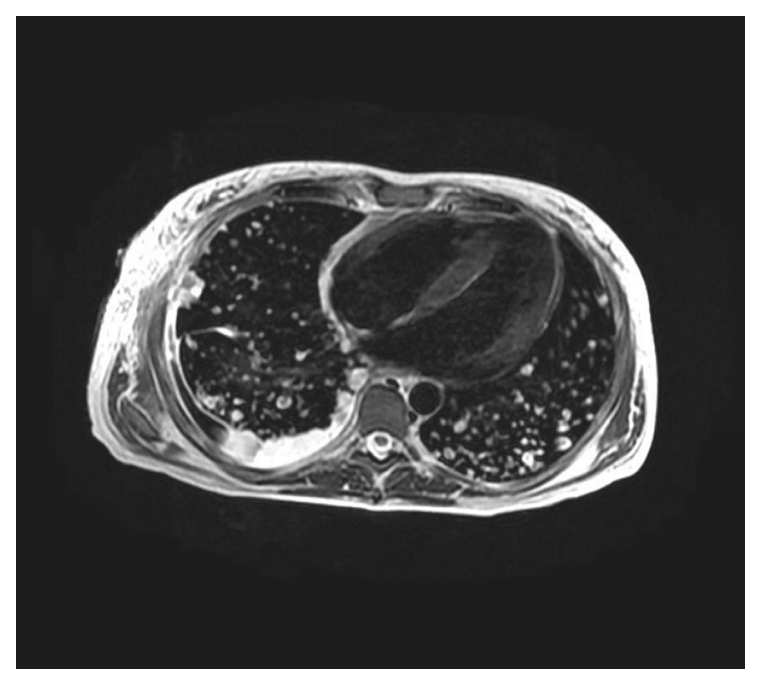

Fig. 1 Magnetic resonance imaging of the thorax, demonstrating bilateral pulmonary nodules (Case 1)
Grocott-Gomori's methenamine silver staining). Culture of BAL fluid (Sabouraud dextrose agar) was also positive for $H$. capsulatum (identification of the mycelial form of $H$. capsulatum at $25^{\circ} \mathrm{C}$ and yeast conversion at $37^{\circ} \mathrm{C}$ ).

Despite the endemic presence of $H$. capsulatum in Latin America, histoplasmosis is unusual in other parts of the world, except for the Mississippi river valley (US). Histoplasmosis seems to be a rare condition after solid organ transplant (SOT) in most parts of the globe. For instance, the Transplant-Associated Infection Surveillance Network (TRANSNET) studied 23 transplant centers in the United States and described the 1-year cumulative incidence of histoplasmosis as $0.1 \%$. Although not frequent in this cohort, histoplasmosis was the most endemic fungal infection seen in the study, with $34 \%$ of cases occurring $>2$ years post transplantation [1]. Moreover, its incidence appears to be increasing: a survey of histoplasmosis-associated hospitalizations in the US revealed an annual percent change in transplant patients of $+12.9 \%$ over $2002-2012$ [2]. It is clear that, in this case, the medical team did not initially think about disseminated histoplasmosis. However, there is plenty of data revealing a high frequency of histoplasmosis in Brazil-in which the southern states and the Amazon are the most affected regions. The patient reported here came from an Amazon state and and the transplant was performed in the south.

Difficulties in achieving an accurate mycological diagnosis in SOT recipients include unspecific clinical syndromes. In transplant recipients, skin lesions (frequent in AIDS patients with disseminated disease) are unusual [3-5]. The absence of respiratory symptoms, as described in this case, made the diagnosis even more challenging. Histoplasmosis seems to follow a more aggressive and rapid course in transplant patients, and clinical awareness is critical for an early diagnosis and intervention.

The differential diagnosis in this patient includes tuberculosis, histoplasmosis, paracoccidioidomycosis, and invasive pulmonary aspergillosis. There is no reliable non-invasive and non-culture-based diagnostic test in this scenario capable of excluding the diseases 
considered in differential diagnosis. Besides the risk associated with the performance of invasive medical procedures (such as the pulmonary biopsy performed in the case), it is to be noted that an experienced pathologist is ultimately needed to correctly identify Histoplasma from histopathologic specimens. This is a small yeast-like fungal pathogen that can easily be overlooked in the hands of an inexperienced physician.

In the microbiology laboratory, Histoplasma may take several weeks to grow, and specific culture media such as Sabouraud dextrose agar or Agar Mycosel are required. Therefore, waiting for culture results may not be the best strategy, since prompt initiation of effective antifungal therapy is essential for this disseminated disease [6].

Histoplasma antigen detection allows for an early diagnosis of disseminated histoplasmosis. These are non-invasive and relatively rapid tests that do not require culture for diagnosis. In SOT recipients, antigen detection is the most important method for the early diagnosis of progressive disseminated histoplasmosis [7, 8]. Antibody detection, as ordered in this case, is of limited use due to its low sensitivity. In a large case series of histoplasmosis in SOT recipients, antigenuria detection was the most sensitive diagnostic method, presented in 93\% of the cases. As expected, antigen was more frequently detected in this study in patients with disseminated histoplasmosis, in comparison to pulmonary disease only (97\% vs. $73 \%$, respectively) [7]. Unfortunately, in countries with the highest burden of disease, access to these diagnostic tests remains very limited. Most tests are based on enzyme immunoassay (ELISA), but point-of-care lateral flow technologies should be available in the near future.

Regarding Histoplasma nucleic acid detection, there is as yet no available commercial kit and few laboratories perform polymerase chain reaction (PCR) tests for the diagnosis of histoplasmosis. Therefore, even though PCR may be a more cost-effective and versatile diagnostic tool in this setting, this review will focus on Histoplasma antigen detection, which is currently the most important test to detect disseminated histoplasmosis.
As shown in this case, it is quite common for patients with histoplasmosis to first receive (inappropriately) therapy against $M$. tuberculosis. This may not only delay diagnosis but also result in toxicities and drug-drug interactions.

In summary, histoplasmosis represents a tough diagnostic challenge, mainly due to the unavailability of modern diagnostic tools like Histoplasma antigen detection, in combination with limited clinical suspicion.

\section{CASE 2}

A 37-year-old man presented with a 2-week story of dry cough and progressive dyspnoea. He had been submitted to an allogeneic hematopoietic cell transplant procedure for a refractory acute myeloid leukemia 4 months before hospital admission. The patient developed grade III graft-versus-host disease (GVHD). He was taking cyclosporine and prednisone $10 \mathrm{mg}$ daily. He received prophylaxis with posaconazole oral solution ( $200 \mathrm{mg}$ daily) in the first 15 days following transplantation (during the neutropenia phase, as per local protocol). A chest computed tomography revealed a bilateral interstitial infiltrate and multiple nodules with a maximum size of $1.3 \mathrm{~cm}$, without halos (Fig. 2). He was non-neutropenic at this time. Bronchoscopy was performed and BAL fluid analysis was negative for fungi (microscopy and culture). The BAL GM index was 0.9. An open lung biopsy revealed

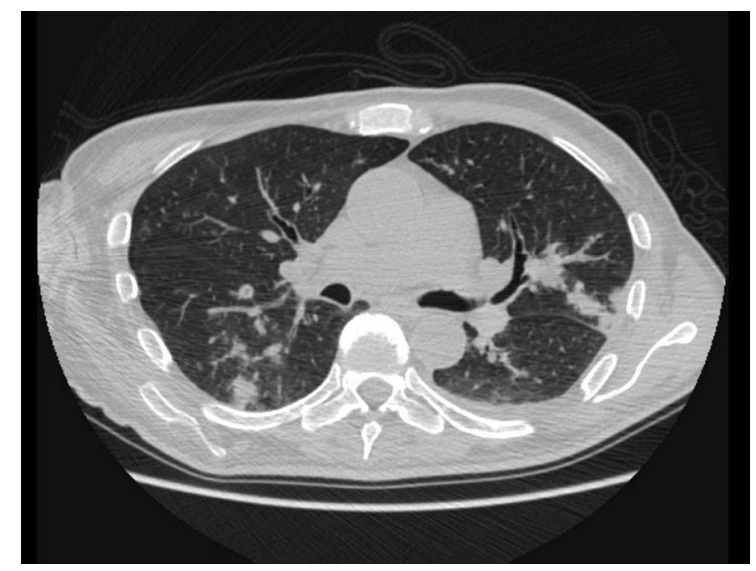

Fig. 2 Computerized tomography of the thorax, showing multiple pulmonary nodules and patchy infiltrates (Case 2) 
invasion by branched hyphae, suggestive of Aspergillus sp. Voriconazole was initiated at standard doses but the patient died 6 days later of respiratory failure and dysfunction of multiple organs and systems. Cultures of the lung specimen were negative.

This was a transplant recipient who had undergone posaconazole prophylaxis and who developed an IFD 3 months after the end of the 30-day prophylactic regimen. Posaconazole prophylaxis represents a safe and effective strategy against fungal infections in transplant patients $[9,10]$. Although there is an ongoing debate regarding the optimal approach to the prevention of IFD in allogeneic stem cell transplant recipients, recent guidelines support the use of prophylactic antifungal agents in these patients, particularly in the context of GVHD $[11,12]$.

There are important differences between aspergillosis in neutropenic hosts and in non-neutropenic hosts [13]. In neutropenic hosts, angioinvasion is a hallmark of the disease and this is associated with a high frequency of dissemination to other organs. In this scenario, classic radiologic findings such as pulmonary nodules and the halo sign are commonly seen. Serum GM, a cell wall component of many fungi including Aspergillus species, can be detected in the sera using a commercial test (Platelia ${ }^{\circledR}$ Aspergillus EIA; BioRad). GM testing is particularly useful for neutropenic patients when serum samples are tested-its high negative predictive value $(>98 \%)$ in this context contributes greatly in the management of these individuals [13-15].

Invasive aspergillosis in the non-neutropenic host is typically not associated with angioinvasion. Symptoms of disease are often unspecific and the clinical course is most often subacute. Typical radiologic hallmarks may also be absent, with a plethora of other pulmonary abnormalities. GM detection in the serum of non-neutropenic hosts is also of limited interest due to its limited sensitivity [16]. However, GM detection in BAL samples is of great utility in non-neutropenic hosts $[17,18]$. The absence of appropriate suspicion of invasive aspergillosis in non-neutropenic patients frequently leads to a delayed diagnosis and a poor outcome, with many cases only diagnosed post-mortem [13].
The case reported here did not represent a breakthrough IFD, since the patient was exposed to posaconazole $>100$ days before developing IFD. However, there are some interesting aspects related to the diagnosis of IFD in patients undergoing antifungal prophylaxis that deserve discussion. We should all bear in mind that GM testing in serum samples has been validated in neutropenic individuals not receiving antifungal prophylaxis against Aspergillus species. In patients receiving anti-Aspergillus antifungal prophylaxis, GM should better be avoided as a tool for preemptive antifungal therapy (due to its reduced test sensitivity in this context) but rather as a diagnostic-driven strategy for patients with a suspected IFD [19].

As for the other azoles, posaconazole has pharmacokinetic properties that require therapeutic drug monitoring (TDM) to assure that serum drug levels are adequate. In prophylactic regimens, however, there is no consensus on the need for serum drug measurements, since posaconazole achieves high tissue concentrations that do not necessarily correlate with serum levels [20]. But considering the formulation and the dose used by our patient, TDM could have been recommended as good clinical practice. Unfortunately, azole drug monitoring is not available in most medical centers in Latin America, even though posaconazole is being widely incorporated in the antifungal armamentarium in transplant centers.

GM testing using a lateral flow device is a new and promising (rapid) diagnostic method with a performance comparable to that of both PCR and classical GM testing (ELISA). Recent data, however, indicate that, in the presence of mold-active antifungal prophylaxis or treatment, sensitivity of this method drops to $52 \%$, thereby compromising its utility in this scenario [21].

Molecular methods like Aspergillus PCR promise to improve diagnosis, but a lack of standardization precludes their wide use [22]. Recently, many efforts have been made in order to standardize Aspergillus PCR [22-24]. There is as yet no consensus on how these tests could be used in the diagnostic strategy, and many issues arise, such as difficulties in the differentiation 
between infection and colonization [23]. A quantitative measurement of Aspergillus DNA could help on this regard, but this remains to be proved [25]. Aspergillus PCR is expected to be included in the next EORTC/MSG definitions of invasive fungal disease $[22,23]$.

Although this review article is devoted to the diagnosis of IFD and not therapy, the authors recognize that treating an IFD with voriconazole in a patient previously exposed to posaconazole is not recommended. Since lung tissue obtained by biopsy from this patient was not cultured, we should refer to this as a case of hyalohyphomycosis (which includes fusariosis and scedosporiosis) and not necessarily invasive aspergillosis. Even though a positive GM test in this context strongly suggests the diagnosis of IA, it is well known that infections caused by Fusarium species may also result in positive GM tests [26]. Treatment with a polyene would be more appropriate in this case, according to ECIL-3 recommendations [27].

\section{CASE 3}

A 58-year-old man reported with a 2-week history of headache and fever. He had been submitted to kidney transplantation 8 months before hospital admission and was taking tacrolimus $(4 \mathrm{mg}$ daily) and prednisone (10 mg daily). Physical examination revealed an erythematous lesion on the right forearm $(10 \mathrm{~cm}$ in diameter), associated with local edema. Chest computed tomography imaging was normal. Serum cryptococcal antigen detection was positive (titer, 1:64; latex agglutination test). Amphotericin B deoxycholate was initiated ( $1 \mathrm{mg} / \mathrm{kg} /$ day). 5-Fluorocytosine (5-FC) was not available. Lumbar puncture revealed a cerebrospinal fluid (CSF) with 12 leucocytes $/ \mathrm{mm}^{3}$; microscopy was negative for fungi and cryptococcal antigen detection was positive (titer, 1:32). After 5 days of antifungal therapy, his clinical condition improved, with regression of skin lesions. Fungal culture in the CSF was negative and the skin lesion was not biopsied.

The most important risk factor for cryptococcosis is HIV infection. Today, HIV infection accounts for up to $80 \%$ of cryptococcal disease globally. However, other populations, such as corticosteroid and/or immunosuppressive therapies including solid organ transplantation recipients, are also at risk $[28,29]$. In a significant parcel of patients, no identifiable risk factor is determined [29]. Cryptococcosis is currently one of the most important opportunistic infections affecting renal transplant recipients [30].

Most cryptococcal infections (95\%) are caused by Cryptococcus neoformans (serotype A), but the other $4-5 \%$ are caused by C.neoformans (serotype D) and C. gattii (serotype B-C). C. gattii has an endemic behavior and is associated to eucalyptus trees in tropical and subtropical climates.

The most common site of extra-pulmonary infection is the central nervous system. In this case, the patient presented with skin lesions. Atypical disease presentations may result in poor outcomes because of delays in diagnosis and treatment; nevertheless, skin lesions are a well-described manifestation in renal transplant patients [28, 30-32]. Skin lesions in cryptococcosis are pleomorphic and a biopsy is crucial for diagnosis, facilitating diagnosis of this potentially lethal condition. Patients using tacrolimus are more likely to develop skin lesions. Tacrolimus is a calcineurin inhibitor and has in vitro activity against Cryptococcus. Cyclosporine A also has anti-cryptococcal activity, but a lower penetration in the CNS. Therefore, these effects could in part explain the higher frequency of extra-neural presentations seen in patients using tacrolimus, particularly when compared to HIV patients [33].

Cryptococcus can be cultured from most sites. In AIDS-related cryptococcal meningitis, the sensitivity of CSF and blood cultures are $~ 90 \%$ and $\sim 50-70 \%$, respectively [34]. Cultures can distinguish between the two species, using a solid agar medium containing canavanine, glycine and bromothymol blue (CGB agar). When $C$. gattii is growing, a positive color change from yellow-green to cobalt blue is noted with CGB $[35,36]$. Even though is a relatively cheap test, CGB is not widely available in most microbiology laboratories around the world.

Cryptococcal antigen detection ( $\mathrm{CrAg}$ ) is a valuable tool for early diagnosis of cryptococcosis. The two main techniques available are 
latex agglutination (LA) and enzyme immunoassay (EIA), performed either in the cerebrospinal fluid or serum [28]. LA sensitivities and specificities vary from 93\% to $100 \%$ and from $93 \%$ to $98 \%$, respectively, regarding the detection of C. neoformans [28]. However, LA has reduced sensitivity for $C$. gattii detection, whilst EIA shows reduced sensitivity for serotype C and D [34]. LA requires laboratory equipment and refrigeration of reagents, and is an ultimately manual test with subjectivity in the interpretation of the result [34]. Most of these characteristics also apply to EIA, with the additional disadvantages of higher cost and turnaround time. Due to these limitations, their use in resource-limited settings is problematic. Nevertheless, these tests have been considered standards of care for over 35 years [28, 34, 37].

Recently, an immune chromatographic lateral flow assay (CrAg LFA; Immuno-Mycologics, Norman, OK, USA) has been designed in order to overcome the need for a rapid and accurate diagnosis, in settings with limited access to laboratory infrastructure. LFA has a sensitivity and specificity $>98 \%$, and a shorter turnaround time in comparison to LA/EIA (only $15 \mathrm{~min}$ ) [28, 34, 37]. LFA can also detect C. gattii, an additional advantage in comparison to the other CrAg test on the market. LFA is also cheaper than its comparators. However, experience with LFA remains limited in transplant patients, including kidney transplant recipients [37].

Therefore, screening for cryptococcal disease may be warranted in transplant patients, with a special attention to kidney transplant recipients. Immunosuppressed patients with skin lesions with any type should always be considered for fungal disease, including cryptococcosis. Antigen testing has a central role in diagnosis, and point-of-care methodologies like LFA need to be appropriately evaluated in this context.

\section{CASE 4}

A 56-year-old diabetic woman with chronic kidney disease under peritoneal dialysis presented with a 2-week history of fever and abdominal pain. Peritoneal fluid analysis revealed 240 leucocytes $/ \mathrm{mm}^{3}$ (60\% were lymphomonocytes). Glucose concentration was normal in the peritoneal fluid, and adenosine deaminase was reduced. No bacteria were detected. She was empirically treated with piperacillin/tazobactam for 8 days, without improvement. The peritoneal catheter was removed and a new peritoneal fluid sample obtained. The catheter was not sent for culture. Fluid analysis demonstrated 200 leucocytes $/ \mathrm{mm}^{3}$ and no bacterial growth. Instead, fungal hyphae were detected and identified as Trichoderma sp., by matrix-assisted laser desorption/ionization time-of-flight mass spectrometry (MALDI-TOF MS). Amphotericin B deoxycholate (0.9 $\mathrm{mg} / \mathrm{kg} / \mathrm{day})$ was initiated and the patient improved clinically. Despite the fungal infection treatment, she died 10 days later as a result of a methicillin-resistant Staphylococcus aureus sepsis.

Here, we describe a rare fungal infection causing peritonitis in a patient on continuous ambulatory peritoneal dialysis (CAPD). Most CAPD-related infections are due to bacteria, mainly Gram-positive rods. Only a small fraction ( $\sim 3-10 \%$ ) are caused by fungi, and the most common fungal etiology is Candida sp. Fungal peritonitis in CAPD patients has a high morbidity and mortality. Fungi have always to be considered as a potential causative agent of CAPD-associated peritonitis when bacterial cultures of peritoneal fluid are negative [38]. Trichoderma sp. is a saprotrophic fungus commonly found in the soil. Trichoderma sp. have been associated with skin infections, and also peritonitis, brain abscess, sinusitis and disseminated infections. Mortality may be as high as 50\% [38].

Considering the detection of hyphae in the peritoneal fluid, correct identification is critical for the adequate therapy. Here, we illustrate the important need for mycology laboratories for proper fungal identification. Trichoderma hyphae appear smooth-walled, hyaline septate and branched, characteristics similar to other agents of hyalohyphomycoses like Aspergillus and Fusarium [38, 39]. Trichoderma can be identified by the colony aspect on Sabouraud dextrose agar and by morphological features of the conidia and phialides. However, cultures may take several days to demonstrate growth and require experienced personnel for 
procedures and correct identification [39]. In this context, MALDI-TOF MS has emerged as a revolutionary tool for rapid identification, differentiation and classification of microorganisms. Filamentous fungi such as Aspergillus, Fusarium and even Trichoderma can be identified using MALDI-TOF. This technology can provide an accurate and rapid diagnose at the species level, which is particularly important in the scenario described, in which classic methods are prone to misidentification and delays [40-42]. Studies have confirmed the discriminatory power and accuracy of MALDI-TOF MS for fungi, overcoming morphological analysis (by time and reliability) and molecular identification (by cost, feasibility and availability) [41, 42]. Unfortunately, MALDI-TOF MS is still not widely available in developing countries like Brazil.

In this case, removal of the dialysis catheter and prompt initiation of antifungal therapy were crucial to patient management. Trichoderma susceptibility to antifungals is variable (which reinforces the need for antifungal susceptibility testing, which was performed in this case). Most isolates of Trichoderma sp. show resistance to fluconazole and 5-FC. Amphotericin B and azoles have variable resistance. Voriconazole have been used, with success, to treat disseminated infections. The role of combination antifungal therapy remains unclear [39].

\section{CASE 5}

A 24-year-old woman with Crohn's disease taking mesalazine (3 g daily) and corticosteroids was hospitalized for a long period due to a perforated intestine and bacterial peritonitis. She was currently taking meropenem and vancomycin and started a new fever and hypotension. Blood cultures were collected and anidulafungin was initiated. Candida orthopsilosis was recovered in blood cultures. After 12 days of treatment, the blood cultures were still positive (six positive cultures in a 12-day interval), and a transoesophageal echocardiography showed a 5 -cm-sized thrombus at the extremity of a central venous catheter, with no vegetation on the cardiac valves. Despite 25 days of antifungal therapy, she remained febrile, with persistent fungemia. Divergences between the medical and surgical teams led to a marked delay in catheter removal. After the catheter was surgically removed, the patient became afebrile within 3 days, with negative blood cultures.

Candidemia is related to many known risk factors, and abdominal surgical procedures (especially those involving hollow viscera) are one of the most important. Our patient was also in the hospital for a long period, and used broad-spectrum antibiotics. This scenario represents a high chance of invasive Candida infection [43].

Invasive Candida infections, particularly candidemia, are on the rise in the hospital scenario $[44,45]$. In an US point-prevalence multicenter study, Candida spp. was the most frequent etiology for primary bloodstream infections [44]. In developing countries such as Brazil, incidence is still increasing, and mortality remains high ( $>40 \%$ ), despite the availability of modern antifungal drugs such as the echinocandins in the antifungal armamentarium [46].

Recently, non-C. albicans species have been identified as a frequent cause of hospital infection. C. parapsilosis is the second most common species isolated from the blood in Latin American countries and the third most common in the US. [47]. Molecular studies demonstrated heterogeneity into this species, and divided it into three groups based in its genetic characteristics. The former $C$. parapsilosis group II was re-designated as C. orthopsilosis [48, 49].

Blood cultures are the gold standard for the diagnosis of candidemia. Nevertheless, this traditional methodology lacks sensitivity (around 50-70\%) and 3-4 days may be required for positive results, characteristics that preclude its reliability in the critically ill patient. Delays in starting antifungal therapy are correlated to a worse prognosis: patients receiving appropriate antifungal therapy on the first day after cultures have an estimated mortality of $24 \%$, increasing to $37 \%$ and $41 \%$, respectively, if therapy is started 1 and 2 days later [50, 51]. 
Beta-D-glucan (BDG), a fungal cell wall component, is a biomarker that has been largely studied for the diagnosis of invasive Candida infections. It was included in the last EORTC/ MSG definitions of invasive fungal infections $[52,53]$. Even though significant heterogeneity exists among studies evaluating the performance of BDG, a meta-analysis found a pooled sensitivity of $76.8 \%$ [95\% confidence interval (CI), 67.1-84.3\%] and $85.3 \%$ specificity (95\% CI 79.6-89.7\%) [53]. Therefore, BDG testing offers a much better sensitivity in comparison to the current gold-standard, blood culture. However, BDG is a high-cost and labour-intensive test, and is currently unavailable in most developing countries. Due to its high negative predictive value, BDG testing has the potential to allow for antifungal therapy to be withheld [54], but the economic impact of such intervention has yet to be determined.

T2 Magnetic Resonance Assay (T2MR) is a promising, novel methodology that uses manual application of magnetic resonance to detect Candida species in whole blood samples, with a turnaround time of less than $3 \mathrm{~h}$. This test has a sensitivity of $91 \%$ and specificity of $98 \%$. The extremely accurate and rapid diagnosis that does not require culture may radically chance the management of invasive Candida infections, with a potential impact on patient mortality [55].

Since C. orthopsilosis is also (as C. parapsilosis) a potent biofilm producer, catheter removal is essential in order to obtain the best clinical outcomes. In the case described here, the catheter was not removed in the first place. This may have led to the formation of biofilm around the device and the persistence of the infection. Catheter removal is a necessity in invasive Candida infections [43] and must always be performed when feasible.

\section{CONCLUSION}

Management of IFDs requires an accurate diagnosis, but unfortunately modern diagnostic tools in the mycology laboratory are scarce in most parts of the world. In this review article, we provide readers with illustrative clinical cases obtained from a developing country, in an attempt to discuss what diagnostic tests should be available, and the advantages and difficulties in incorporating such novel technologies.

\section{ACKNOWLEDGEMENTS}

No funding or sponsorship was received for this study or publication of this article. All named authors meet the International Committee of Medical Journal Editors (ICMJE) criteria for authorship for this manuscript, take responsibility for the integrity of the work as a whole, and have given final approval for the version to be published.

Disclosures. Dr. Falci has received research support from United Medical, Bagó Laboratories (now TEVA); has received travel Grants from Gilead, Astellas, MSD, Pfizer and United Medical; and has given lectures for United Medical and Pfizer. Dr. Stadnik has no potential conflicts of interest to declare. In the past 5 years, $\mathrm{Dr}$ Pasqualotto has received research Grants from MSD, Pfizer, United Medical, and Gilead. He has given paid talks on behalf of Astellas, Gilead, United Medical, Pfizer, and MSD.

Compliance with Ethics Guidelines. Informed consent was obtained from all patients for being included in the study.

Open Access. This article is distributed under the terms of the Creative Commons Attribution-NonCommercial 4.0 International License (http://creativecommons.org/licenses/ by-nc/4.0/), which permits any noncommercial use, distribution, and reproduction in any medium, provided you give appropriate credit to the original author(s) and the source, provide a link to the Creative Commons license, and indicate if changes were made.

\section{REFERENCES}

1. Kauffman CA, Freifeld AG, Andes DR, Baddley JW, Herwaldt L, Walker RC, et al. Endemic fungal 
infections in solid organ and hematopoietic cell transplant recipients enrolled in the Transplant-Associated Infection Surveillance Network (TRANSNET). Transpl Infect Dis. 2014;16(2):213-24. doi:10.1111/tid.12186.

2. Benedict K, Derado G, Mody RK. Histoplasmosis-associated hospitalizations in the United States, 2001-2012. Open Forum Infect Dis. 2016;3(1):ofv219. doi:10.1093/ofid/ofv219.

3. Cunha VS, Zampese MS, Aquino VR, Cestari TF, Goldani LZ. Mucocutaneous manifestations of disseminated histoplasmosis in patients with acquired immunodeficiency syndrome: particular aspects in a Latin-American population. Clin Exp Dermatol. 2007;32(3):250-5. doi:10.1111/j.1365-2230.2007. 02392.x.

4. Freifeld AG, Iwen PC, Lesiak BL, Gilroy RK, Stevens RB, Kalil AC. Histoplasmosis in solid organ transplant recipients at a large Midwestern university transplant center. Transpl Infect Dis. 2005;7(3-4):109-15. doi:10.1111/j.1467-8365.2005. 00105.x.

5. Goldani LZ, Aquino VR, Lunardi LW, Cunha VS, Santos RP. Two specific strains of Histoplasma capsulatum causing mucocutaneous manifestations of histoplasmosis: preliminary analysis of a frequent manifestation of histoplasmosis in southern Brazil. Mycopathologia. 2009;167(4):181-6. doi:10.1007/ s11046-008-9171-7.

6. Wheat J, French ML, Kohler RB, Zimmerman SE, Smith WR, Norton JA, et al. The diagnostic laboratory tests for histoplasmosis: analysis of experience in a large urban outbreak. Ann Intern Med. 1982;97(5):680-5.

7. Assi M, Martin S, Wheat LJ, Hage C, Freifeld A, Avery R, et al. Histoplasmosis after solid organ transplant. Clin Infect Dis. 2013;57(11):1542-9. doi:10.1093/cid/cit593.

8. Cuellar-Rodriguez J, Avery RK, Lard M, Budev M, Gordon SM, Shrestha NK, et al. Histoplasmosis in solid organ transplant recipients: 10 years of experience at a large transplant center in an endemic area. Clin Infect Dis. 2009;49(5):710-6. doi:10. $1086 / 604712$.

9. Cornely OA, Maertens J, Winston DJ, Perfect J, Ullmann AJ, Walsh TJ, et al. Posaconazole vs Fluconazole or itraconazole prophylaxis in patients with neutropenia. New Engl J Med. 2007;356(4):348-59. doi:10.1056/NEJMoa061094.

10. Ullmann AJ, Lipton JH, Vesole DH, Chandrasekar P, Langston A, Tarantolo SR, et al. Posaconazole or fluconazole for prophylaxis in severe graft-versus-host disease. New Engl J Med. 2007;356(4):335-47. doi:10.1056/NEJMoa061098.

11. Chowdhary A, Girmenia C, Annino L, Mariotti B, Fanci R, Minotti C et al. Posaconazole oral suspension primary prophylaxis in acute leukemia and allogeneic stem cell transplant patients: can it be used without measurement of plasma concentration? Mycoses. 2016;54(5):445-58. doi:10.1111/ myc.12519, doi:10.1093/mmy/myw001.

12. Winston DJ, Bartoni K, Territo MC, Schiller GJ. Efficacy, safety, and breakthrough infections associated with standard long-term posaconazole antifungal prophylaxis in allogeneic stem cell transplantation recipients. Biol Blood Marrow Transpl J Am Soc Blood Marrow Transpl. 2011;17(4):507-15. doi:10.1016/j.bbmt.2010.04. 017.

13. Kosmidis C, Denning DW. Republished: the clinical spectrum of pulmonary aspergillosis. Postgrad Med J. 1077;2015(91):403-10. doi:10.1136/ postgradmedj-2014-206291rep.

14. Leeflang MM, Debets-Ossenkopp YJ, Wang J, Visser CE, Scholten RJ, Hooft L, et al. Galactomannan detection for invasive aspergillosis in immunocompromised patients. Cochrane Database Syst Rev. 2015;12:CD007394. doi:10.1002/14651858. CD007394.pub2.

15. Maertens J, Verhaegen J, Demuynck $H$, Brock $P$, Verhoef G, Vandenberghe P, et al. Autopsy-controlled prospective evaluation of serial screening for circulating galactomannan by a sandwich enzyme-linked immunosorbent assay for hematological patients at risk for invasive Aspergillosis. J Clin Microbiol. 1999;37(10):3223-8.

16. Cordonnier C, Botterel F, Ben Amor R, Pautas C, Maury S, Kuentz M, et al. Correlation between galactomannan antigen levels in serum and neutrophil counts in haematological patients with invasive aspergillosis. Clin Microbiol Infect. 2009;15(1):81-6. doi:10.1111/j.1469-0691.2008. 02122.x.

17. Pasqualotto AC, Xavier MO, Sanchez LB, de Oliveira Costa CD, Schio SM, Camargo SM, et al. Diagnosis of invasive aspergillosis in lung transplant recipients by detection of galactomannan in the bronchoalveolar lavage fluid. Transplantation. 2010;90(3):306-11. doi:10.1097/TP. ob013e3181e49bc1.

18. Zou M, Tang L, Zhao S, Zhao Z, Chen L, Chen P, et al. Systematic review and meta-analysis of detecting galactomannan in bronchoalveolar lavage fluid for diagnosing invasive aspergillosis. PLoS ONE. 2012;7(8):e43347. doi:10.1371/journal. pone.0043347. 
19. Duarte RF, Sanchez-Ortega I, Cuesta I, Arnan M, Patino B, Fernandez de Sevilla A, et al. Serum galactomannan-based early detection of invasive aspergillosis in hematology patients receiving effective antimold prophylaxis. Clin Infect Dis. 2014;59(12):1696-702. doi:10.1093/cid/ciu673.

20. Girmenia C, Annino L, Mariotti B, Fanci R, Minotti C, Spadea A, et al. Posaconazole oral suspension primary prophylaxis in acute leukemia and allogeneic stem cell transplant patients: can it be used without measurement of plasma concentration? Med Mycol. 2016;54(5):445-58. doi:10.1093/mmy/ myw001.

21. Eigl S, Prattes J, Reinwald M, Thornton CR, Reischies F, Spiess B, et al. Influence of mould-active antifungal treatment on the performance of the Aspergillus-specific bronchoalveolar lavage fluid lateral-flow device test. Int J Antimicrob Agents. 2015;46(4):401-5. doi:10.1016/j.ijantimicag.2015. 05.017 .

22. White PL, Wingard JR, Bretagne S, Loffler J, Patterson TF, Slavin MA, et al. Aspergillus polymerase chain reaction: systematic review of evidence for clinical use in comparison with antigen testing. Clin Infect Dis. 2015;61(8):1293-303. doi:10.1093/ cid/civ507.

23. Pasqualotto AC, Falci DR. Has Aspergillus PCR come to the age of maturity? Mycopathologia. 2016;181(9-10):623-4. doi:10.1007/s11046-0160033-4.

24. Springer J, White PL, Hamilton S, Michel D, Barnes $\mathrm{RA}$, Einsele $\mathrm{H}$, et al. Comparison of performance characteristics of Aspergillus PCR in testing a range of blood-based samples in accordance with international methodological recommendations. J Clin Microbiol. 2016;54(3):705-11. doi:10.1128/jcm. 02814-15.

25. Zhang S, Wang S, Wan Z, Que C, Li R, Yu J. Quantitative real-time PCR and platelia galactomannan assay for the diagnosis of invasive pulmonary aspergillosis: bronchoalveolar lavage fluid performs better than serum in non-neutropaenic patients. Mycopathologia. 2016;181(9-10):625-9. doi:10.1007/s11046-016-0024-5.

26. Nucci M, Carlesse F, Cappellano P, Varon AG, Seber A, Garnica M, et al. Earlier diagnosis of invasive fusariosis with Aspergillus serum galactomannan testing. PLoS ONE. 2014;9(1):e87784. doi:10.1371/ journal.pone.0087784.

27. Castagna L, Bramanti S, Sarina B, Todisco E, Ibatici A, Santoro A. ECIL 3-2009 update guidelines for antifungal management. Bone Marrow Transpl. 2012;47(6):866. doi:10.1038/bmt.2011.154.
28. Maziarz EK, Perfect JR. Cryptococcosis. Infect Dis Clin N Am. 2016;30(1):179-206. doi:10.1016/j.idc. 2015.10.006.

29. Pappas PG, Perfect JR, Cloud GA, Larsen RA, Pankey GA, Lancaster DJ, et al. Cryptococcosis in human immunodeficiency virus-negative patients in the era of effective azole therapy. Clin Infect Dis. 2001;33(5):690-9. doi:10.1086/322597.

30. Badiee P, Alborzi A. Invasive fungal infections in renal transplant recipients. Exp Clin Transpl. 2011;9(6):355-62.

31. Orsini J, Nowakowski J, Delaney V, Sakoulas G, Wormser GP. Cryptococcal infection presenting as cellulitis in a renal transplant recipient. Transpl Infect Dis. 2009;11(1):68-71. doi:10.1111/j.13993062.2008.00352.x.

32. Pasqualotto AC, Bittar AE, de Quadros M, Severo LC. Cryptococcal cellulitis in a renal transplant patient. Nephrol Dial Transpl. 2005;20(9):2007-8. doi:10.1093/ndt/gfh801.

33. Singh N, Alexander BD, Lortholary O, Dromer F, Gupta KL, John GT, et al. Cryptococcus neoformans in organ transplant recipients: impact of calcineurin-inhibitor agents on mortality. J Infect Dis. 2007;195(5):756-64. doi:10.1086/511438.

34. Vidal JE, Boulware DR. Lateral flow assay for cryptococcal antigen: an important advance to improve the continuum of hiv care and reduce cryptococcal meningitis-related mortality. Rev Inst Med Trop Sao Paulo. 2015;57(Suppl 19):38-45. doi:10.1590/ s0036-46652015000700008.

35. Kwon-Chung KJ, Polacheck I, Bennett JE. Improved diagnostic medium for separation of Cryptococcus neoformans var. neoformans (serotypes A and D) and Cryptococcus neoformans var. gattii (serotypes B and C). J Clin Microbiol. 1982;15(3):535-7.

36. Polacheck I, Kwon-Chung KJ. Canavanine resistance in Cryptococcus neoformans. Antimicrob Agents Chemother. 1986;29(3):468-73.

37. Jarvis JN, Percival A, Bauman S, Pelfrey J, Meintjes G, Williams GN, et al. Evaluation of a novel point-of-care cryptococcal antigen test on serum, plasma, and urine from patients with HIV-associated cryptococcal meningitis. Clin Infect Dis. 2011;53(10):1019-23. doi:10.1093/cid/cir613.

38. Esel D, Koc AN, Utas C, Karaca N, Bozdemir N. Fatal peritonitis due to Trichoderma sp. in a patient undergoing continuous ambulatory peritoneal dialysis. Mycoses. 2003;46(1-2):71-3.

39. Festuccia M, Giaccone L, Gay F, Brunello L, Maffini E, Ferrando F, et al. Trichoderma species fungemia 
after high-dose chemotherapy and autologous stem cell transplantation: a case report. Transpl Infect Dis. 2014;16(4):653-7. doi:10.1111/tid.12245.

40. Chalupova J, Raus M, Sedlarova M, Sebela M. Identification of fungal microorganisms by MALDI-TOF mass spectrometry. Biotechnol Adv. 2014;32(1):230-41. doi:10.1016/j.biotechadv.2013. 11.002 .

41. Sanguinetti M, Posteraro B. MALDI-TOF mass spectrometry: any use for Aspergilli? Mycopathologia. 2014;178(5-6):417-26. doi:10.1007/ s11046-014-9757-1.

42. De Carolis E, Posteraro B, Lass-Florl C, Vella A, Florio AR, Torelli R, et al. Species identification of Aspergillus, Fusarium and Mucorales with direct surface analysis by matrix-assisted laser desorption ionization time-of-flight mass spectrometry. Clin Microbiol Infect. 2012;18(5):475-84. doi:10.1111/j. 1469-0691.2011.03599.x.

43. Pappas PG, Kauffman CA, Andes DR, Clancy CJ, Marr KA, Ostrosky-Zeichner L, et al. Clinical practice guideline for the management of candidiasis: 2016 update by the infectious diseases Society of America. Clin Infect Dis. 2016;62(4):e1-50. doi:10. 1093/cid/civ933.

44. Magill SS, Edwards JR, Bamberg W, Beldavs ZG, Dumyati G, Kainer MA, et al. Multistate point-prevalence survey of health care-associated infections. New Engl J Med. 2014;370(13):1198-208. doi:10.1056/ NEJMoa1306801.

45. Martin GS, Mannino DM, Eaton S, Moss M. The epidemiology of sepsis in the United States from 1979 through 2000. New Engl J Med. 2003;348(16):1546-54. doi:10.1056/ NEJMoa022139.

46. Doi AM, Pignatari AC, Edmond MB, Marra AR, Camargo LF, Siqueira RA, et al. Epidemiology and microbiologic characterization of nosocomial candidemia from a brazilian national surveillance program. PLoS ONE. 2016;11(1):e0146909. doi:10. 1371/journal.pone.0146909.

47. Pfaller MA, Jones RN, Doern GV, Sader HS, Messer SA, Houston A, et al. Bloodstream infections due to
Candida species: sENTRY antimicrobial surveillance program in North America and Latin America, 1997-1998. Antimicrob Agents Chemother. 2000;44(3):747-51.

48. Constante CC, Monteiro AA, Alves SH, Carneiro LC, Machado MM, Severo LC, et al. Different risk factors for candidemia occur for Candida species belonging to the C. parapsilosis complex. Med Mycol. 2014;52(4):403-6. doi:10.1093/mmy/myt034.

49. Roy B, Meyer SA. Confirmation of the distinct genotype groups within the form species Candida parapsilosis. J Clin Microbiol. 1998;36(1):216-8.

50. Garey KW, Rege M, Pai MP, Mingo DE, Suda KJ, Turpin RS, et al. Time to initiation of fluconazole therapy impacts mortality in patients with candidemia: a multi-institutional study. Clin Infect Dis. 2006;43(1):25-31. doi:10.1086/504810.

51. Ericson EL, Klingspor L, Ullberg M, Ozenci V. Clinical comparison of the Bactec Mycosis IC/F, BacT/Alert FA, and BacT/Alert FN blood culture vials for the detection of candidemia. Diagn Microbiol Infect Dis. 2012;73(2):153-6. doi:10. 1016/j.diagmicrobio.2012.02.020.

52. Denning DW, Perlin DS, Muldoon EG, Colombo AL, Chakrabarti A, Richardson MD, et al. Delivering on antimicrobial resistance agenda not possible without improving fungal diagnostic capabilities. Emerg Infect Dis. 2017. doi:10.3201/eid2302. 152042 .

53. Karageorgopoulos DE, Vouloumanou EK, Ntziora F, Michalopoulos A, Rafailidis PI, Falagas ME. beta-D-glucan assay for the diagnosis of invasive fungal infections: a meta-analysis. Clin Infect Dis. 2011;52(6):750-70. doi:10.1093/cid/ciq206.

54. Nucci M, Nouér SA, Esteves P, Guimarães T, Breda $G$, de Miranda BG et al. Discontinuation of empirical antifungal therapy in ICU patients using 1 , 3- $\beta$-D-glucan. J Antimicrob Chemother. 2016;71(9):2628-33.

55. Mylonakis E, Clancy CJ, Ostrosky-Zeichner L, Garey KW, Alangaden GJ, Vazquez JA, et al. T2 magnetic resonance assay for the rapid diagnosis of candidemia in whole blood: a clinical trial. Clin Infect Dis. 2015;60(6):892-9. doi:10.1093/cid/ciu959. 